\title{
AS602801, an Anticancer Stem Cell Candidate Drug, Reduces Survivin Expression and Sensitizes A2780 Ovarian Cancer Stem Cells to Carboplatin and Paclitaxel
}

\author{
MASAHIRO YAMAMOTO ${ }^{1}$, SHUHEI SUZUKI ${ }^{1,2}$, KEITA TOGASHI $^{1,3}$, TOMOMI SANOMACHI ${ }^{1,2}$, \\ SHIZUKA SEINO ${ }^{1}$, CHIFUMI KITANAKA ${ }^{1,4}$ and MASASHI OKADA ${ }^{1}$ \\ Departments of ${ }^{1}$ Molecular Cancer Science, ${ }^{2}$ Clinical Oncology and ${ }^{3}$ Ophthalmology, \\ Yamagata University School of Medicine, Yamagata, Japan; \\ ${ }^{4}$ Research Institute for Promotion of Medical Sciences, Yamagata University Faculty of Medicine, Yamagata, Japan
}

\begin{abstract}
Background: AS602801, a novel inhibitor of c-Jun $N$-terminal kinase (JNK), suppresses tumor initiation capacity and metastatic potential of cancer stem cells (CSCs). However, it remains unknown whether this inhibitor can chemosensitize CSCs. Materials and Methods: Using A2780 CSLC, a CSC line derived from ovarian cancer, this study examined the combinational effects of AS602801 and carboplatin or paclitaxel and explored the mechanism of those effects. Results: AS602801 chemosensitized A2780 CSLC cells to carboplatin and paclitaxel. With respect to the mechanism of chemosensitization, the expression of survivin, an anti-apoptotic protein, was reduced by AS602801. Pharmacological and genetic inhibition of survivin chemosensitized the cells to carboplatin and paclitaxel. Suppression of survivin by AS602801 was also observed in other types of CSCs and nonCSCs. Conclusion: AS602801, which reduces survivin expression, can chemosensitize ovarian CSCs and is a candidate drug that targets the chemoresistance, tumorinitiating capacity and metastasis of CSCs.
\end{abstract}

Cancer stem cells (CSCs) are rare populations in tumor tissue and have high tumor initiation capacity and chemoresistance. Because of these properties, CSCs are considered to be major sources of frequent recurrence after chemotherapy in various types of cancers $(1,2)$. For instance, in ovarian cancer, although standard first-line chemotherapy, a combination of platinum and taxanes,

Correspondence to: Masahiro Yamamoto and Masashi Okada, Department of Molecular Cancer Science, Yamagata University School of Medicine, Yamagata, 990-9585, Japan. Tel: +81 236285214, Fax: +81 236285215, e-mail: masahiro@med.id.yamagata-u.ac.jp (M.Y.),m-okada@med.id.yamagata-u.ac.jp (M.O.)

Key Words: Drug repositioning, repurposing, drug resistance. demonstrates a high response rate, recurrence at local and distant sites frequently occurs (3-5), and CSCs are considered to play a role in frequent recurrence $(6,7)$. Eliminating CSCs is important for suppressing recurrence and improving prognosis of cancer. Therefore, CSCs are considered promising targets of cancer chemotherapy (8).

We reported that AS602801, an inhibitor of c-Jun $\mathrm{N}$-terminal kinase (JNK), is a novel drug that inhibits the tumor-initiating capacity of CSCs (9). We also reported that AS602801 has the potential to inhibit metastasis via the suppression of cell-cell communication between CSCs and parenchymal cells at metastatic sites (10). Furthermore, the safety of AS602801 has already been assessed in patients with endometriosis in a phase II clinical trial (NCT 01630252). Therefore, AS602801 is considered a good candidate to partner with standard chemotherapeutic agents in cancer. However, combinational effects of AS602801 with a standard chemotherapy in CSCs remain unknown. In this study, we investigated whether and how AS602801 sensitizes CSCs to chemotherapeutic reagents using ovarian CSCs.

\section{Materials and Methods}

Antibodies and reagents. Antibodies against cellular inhibitor of apoptosis 1 (c-IAP1), c-IAP2, X-linked inhibitor of apoptosis protein (XIAP), survivin, livin, B-cell lymphoma 2 (BCL-2)-like protein 11 (BIM), and glyceraldehyde 3-phosphate dehydrogenase (GAPDH) were purchased from Cell Signaling Technology, Inc. (Danvers, MA, USA). An antibody against BCL-2-associated X protein (BAX) was purchased from Becton, Dickinson and Company (Franklin Lakes, NJ, USA). Antibodies against BCL-2 antagonist/killer (BAK), BCL-2-related ovarian killer (BOK), BCL-2-associated death promoter (BAD), BH3 interacting-domain death agonist (BID), p53 upregulated modulator of apoptosis (PUMA), BCL-2, myeloid cell leukemia-1 (MCL1), and BCL-xL were obtained from Santa Cruz Biotechnology, Inc. (Santa Cruz, CA, USA). AS602801 and YM155, a small-molecule suppressant of survivin, were purchased from ChemScene (Monmouth Junction, NJ, USA) and from AdooQ 
BioScience (Irvine, CA, USA), respectively. These drugs were dissolved in dimethyl sulfoxide (DMSO) to prepare a $10 \mathrm{mM}$ and 20 $\mu \mathrm{M}$ stock solution, respectively. Carboplatin was purchased from FUJIFILM Wako Pure Chemical (Osaka, Japan) and dissolved in distilled water to prepare a $25 \mathrm{mM}$ stock solution. Paclitaxel was purchased from TOCRIS Bioscience (Bristol, UK) and dissolved in DMSO to prepare a $10 \mathrm{mM}$ stock solution.

Cell culture. The establishment and characterization of the human CSCs used in this study (A2780 CSLC, TOV-21G CSLC, PANC-1 CSLC, A549 CSLC, and GS-Y03) have been described elsewhere (9, 11-15). These cells were maintained as monolayers under appropriate stem cell culture conditions. Briefly, the cells were cultured on collagen I-coated dishes (IWAKI, Tokyo, Japan) in stem cell culture medium [Dulbecco's modified Eagle's medium (DMEM)/F12 medium supplemented with 1\% B27 (Thermo Fisher Scientific, Waltham, MA, USA), $20 \mathrm{ng} / \mathrm{ml}$ epidermal growth factor (EGF) and fibroblast growth factor 2 (FGF2; Peprotech, Inc., Rocky Hill, NJ, USA), D-(+)-glucose (final concentration=26.2 $\mathrm{mM}$ ), L-glutamine (final concentration $=4.5 \mathrm{mM}$ ), $100 \mathrm{units} / \mathrm{ml}$ penicillin, and $100 \mu \mathrm{g} / \mathrm{ml}$ streptomycin]. The stem cell culture medium was changed approximately every 3 days, and EGF and FGF2 were added to the culture medium every day. TOV-21G was maintained in DMEM/F12. A549 and H1299 were maintained in DMEM. DMEM/F12 and DMEM were supplemented with $10 \%$ fetal bovine serum, $100 \mathrm{units} / \mathrm{ml}$ penicillin, and $100 \mu \mathrm{g} / \mathrm{ml}$ streptomycin. The authenticity of the cells was verified by genotyping short tandem repeat (STR) loci (Bio-Synthesis, Inc., Lewisville, TX, USA) followed by comparison to the American Type Culture Collection STR Database for Human Cell Lines (http://www.atcc.org/STR_Database.aspx).

Gene silencing by siRNA. siRNAs against human survivin and MISSION $^{\circledR}$ siRNA Negative Control were purchased from Thermo Fisher Scientific and Millipore Sigma (St. Louis, MO, USA), respectively. Briefly, A2780 CSLCs were transiently transfected with siRNAs against survivin (siSurvivin) or with a control siRNA (siControl) and were treated on the next day with or without $5 \mu \mathrm{M}$ carboplatin or $2 \mathrm{nM}$ paclitaxel for 3 days. The cells were then subjected to immunoblot analysis of survivin and to a cell death assay using propidium iodide (PI). Transfection of siRNAs was performed using Lipofectamine RNAiMAX (Thermo Fisher Scientific) according to the manufacturer's instructions.

Immunoblot analysis. Immunoblot analysis was conducted as previously described $(9,11-14)$. Cells were washed with ice-cold phosphate-buffered saline and lysed in RIPA buffer [10 mM Tris/HCl ( $\mathrm{pH}$ 7.4), $0.1 \%$ sodium dodecyl sulfate (SDS), $1.5 \mathrm{mM} \mathrm{Na}_{3} \mathrm{VO}_{4}, 10$ $\mathrm{mM} \mathrm{NaF}, 10 \mathrm{mM}$ sodium pyrophosphate, $10 \mathrm{mM}$ sodium $\beta$ glycerophosphate and $1 \%$ protease inhibitor cocktail set III (FUJIFILM Wako Pure Chemical)]. This was followed by the immediate addition of the same volume of Laemmli buffer $2 \times[125$ $\mathrm{mM}$ Tris $/ \mathrm{HCl}$ (pH 6.8), $4 \%$ SDS, $10 \%$ glycerol] and boiling at $95^{\circ} \mathrm{C}$ for $10 \mathrm{~min}$. The protein concentration was determined using a BCA protein assay kit (Pierce Biotechnology, Inc., Rockford, IL, USA). Samples containing equal amounts of protein were resolved by SDSpolyacrylamide gel electrophoresis and transferred to polyvinylidene fluoride membranes. The membranes were probed with a primary antibody against BAX, BAK, BOK, BIM, BAD, BID, PUMA, BCL2, MCL-1, BCL-xL, c-IAP1, c-IAP2, XIAP, survivin, livin, or GAPDH and subsequently with a horseradish peroxidase (HRP)- conjugated secondary antibody as recommended by the manufacturer of each antibody. Specific bands were visualized using Immobilon Western Chemiluminescent HRP Substrate (Merck Millipore, Billerica, MA, USA) and detected semi-quantitatively by a ChemiDoc Touch Imaging System (Bio-Rad, Hercules, CA, USA).

Cell death assay. The cell death assay was performed as previously described $(9,11,14)$. Briefly, cells were incubated in situ with PI $(1 \mu \mathrm{g} / \mathrm{ml})$ and Hoechst $33342(10 \mu \mathrm{g} / \mathrm{ml})$ for $5 \mathrm{~min}$ at $37^{\circ} \mathrm{C}$ in a $\mathrm{CO}_{2}$ incubator to stain dead cells and cell nuclei, respectively. Then the numbers of PI-and Hoechst-positive cells were scored under a fluorescence microscope (CKX41; OLYMPUS, Tokyo, Japan), and the percentage of PI-positive cells (dead cells) relative to Hoechstpositive cells (total cells) was determined. Cell death assays were performed with four or six replicates.

Cell viability assay. The cell viability assay was performed as previously described (11). Briefly, cell viability was determined by the tetrazolium salt reduction method using WST-8 (Cell Counting Kit-8; Dojindo Laboratories, Kumamoto, Japan) according to the manufacturer's instructions. Cells (500 cells/well) plated in 96-well collagen I-coated plates were treated on the next day with $5 \mu \mathrm{M}$ carboplatin or $2 \mathrm{nM}$ paclitaxel in the absence or presence of 7.5 $\mu \mathrm{M}$ AS602801 or $10 \mathrm{nM}$ YM155 for 3 days, followed by culture in the absence of any drug for another 3 days. WST- 8 reagent was then added, and the cells were incubated for $1-3 \mathrm{~h}$ at $37^{\circ} \mathrm{C}$. Absorbance at $450 \mathrm{~nm}$ was measured using a microplate reader (Model 680, Bio-Rad). Relative cell viability was calculated as a percentage of the absorbance of treated samples relative to that of the control samples. Cell viability assays were performed with four or six replicates.

Colony-formation assay. Colony-formation assay was performed as described previously (16). In brief, cells were seeded at a low, colony-forming density (500 cells $/ 35-\mathrm{mm}$ dish) and cultured with $7.5 \mu \mathrm{M}$ AS602801, $5 \mu \mathrm{M}$ carboplatin, $2 \mathrm{nM}$ paclitaxel, or combination of them for 3 days. After cultured for $\sim 1$ week without any drug, the cells were fixed with paraformaldehyde $(4 \% \mathrm{v} / \mathrm{v})$, followed by staining with crystal violet $(0.1 \% \mathrm{w} / \mathrm{v})$.

Statistical analysis. The results were expressed as the mean and standard deviation (SD), and differences were compared using oneway analysis of variance (ANOVA) with Tukey's post-hoc test. Differences with $p$-values of less than 0.05 were considered statistically significant.

\section{Results}

AS602801 can sensitize A2780 ovarian CSCs to carboplatin and paclitaxel. To investigate the combinational effects of AS602801 with chemotherapeutic agents in CSCs, a CSC line derived from the ovarian cancer cell line A2780, A2780 CSLC, was used. The percentage of dead cells was evaluated 3 days after treatment with either carboplatin or paclitaxel and a combination of both with AS602801, using double staining with PI (to stain dead cells) and Hoechst 33342 (to stain both live and dead cells) (Figure 1A). Compared with the treatment with either drug alone, the combination of AS602801 with carboplatin or paclitaxel significantly 


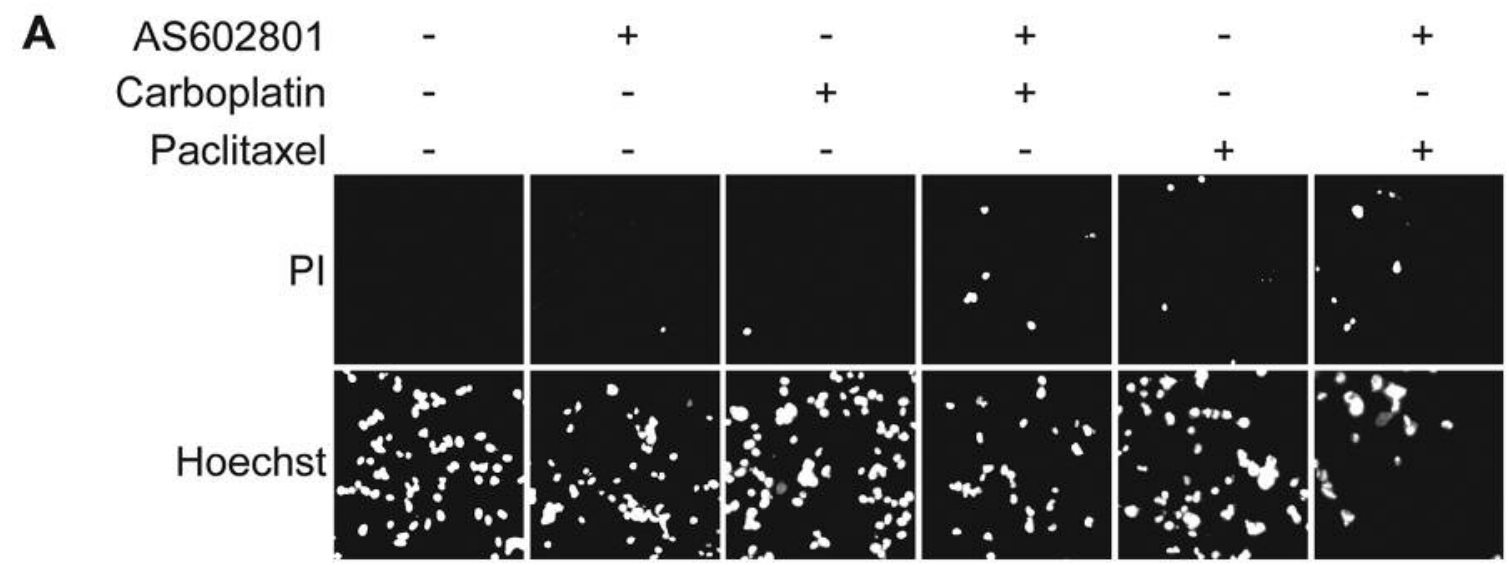

B

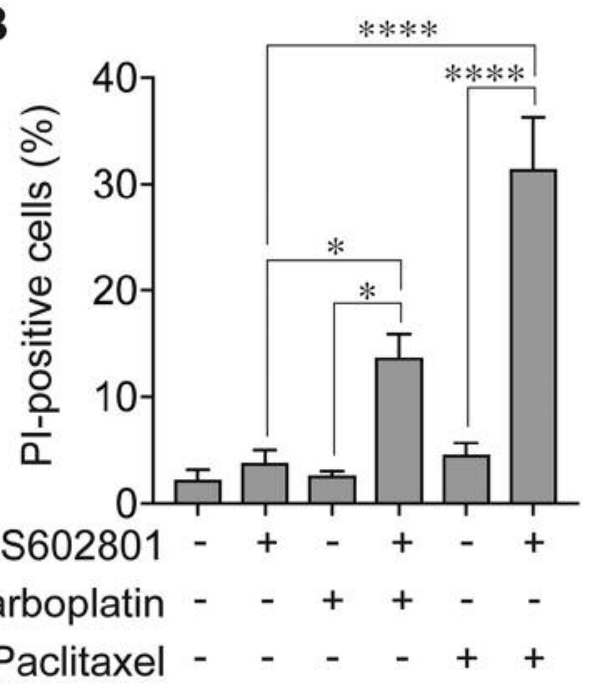

D

\section{Carboplatin}

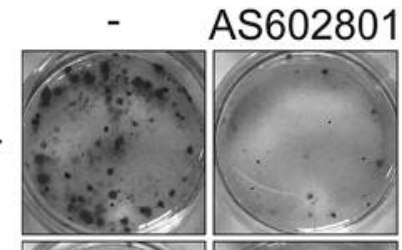

Paclitaxel

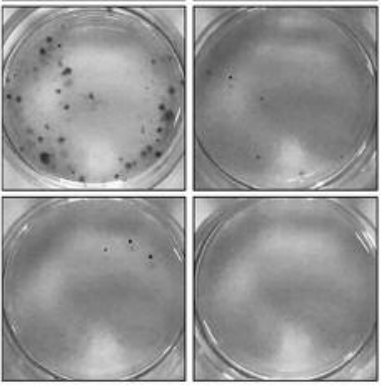

C

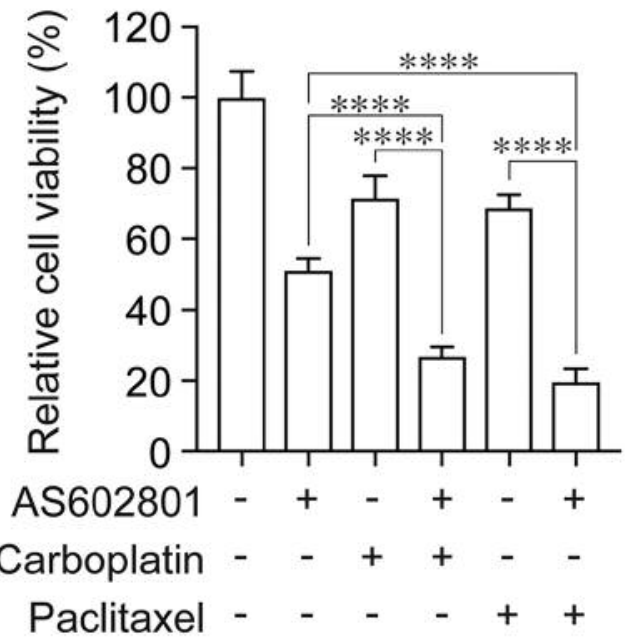

$\mathbf{E}$

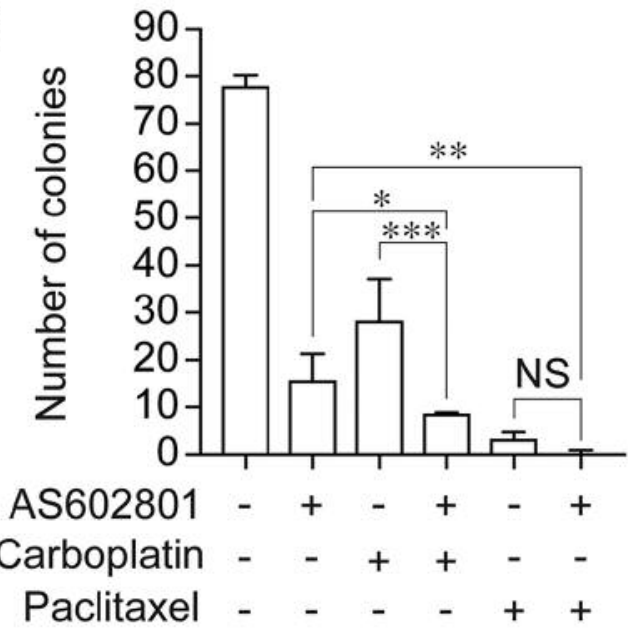

Figure 1. In combination with carboplatin or paclitaxel, AS602801 exerts additional inhibitory effects on A2780 CSLC cells. A2780 CSLC cells treated with or without carboplatin $(5 \mu M)$ or paclitaxel $(2 \mathrm{nM})$ in the absence or presence of AS602801 $(7.5 \mu \mathrm{M})$ for 3 days were subjected to a cell death assay using propidium iodide (PI) as a vital dye. Representative images (A) and quantification of percentage of PI-positive cells (B) are shown. A2780 CSLC cells treated with or without carboplatin $(5 \mu \mathrm{M})$ or paclitaxel $(2 \mathrm{nM})$ in the absence or presence of AS602801 $(7.5 \mu \mathrm{M})$ for 3 days. The cells were then cultured for another 3 days and subjected to a cell viability assay using WST-8 (C), or another 6 days and colonies were visualized by crystal violet stain $(D)$ then the number of colonies were quantified $(E)$. Values represent means $+S D$ from samples counted in four $(E)$ or six $(B, C)$ replicates of a representative experiment repeated with similar results. NS: Non-significant, $p>0.05$; significantly different at: $* p<0.05, * * p<0.01, * * * p<0.001$, and $* * * * p<0.0001$. 
increased cell death (Figure 1B). The viability of cells was also evaluated by the WST- 8 assay which showed that the combination of AS602801 with carboplatin or paclitaxel reduced cell viability significantly more effectively compared to either treatment alone (Figure 1C). Furthermore, in order to determine whether the combinational effects of AS602801 can be translated into long-term inhibition of clonogenic survival, a colony formation assay was performed. The combination of AS602801 with carboplatin significantly reduced the number of colonies formed compared with either treatment alone. Although not statistically significant, the combination of AS602801 and paclitaxel tended to reduce the number of colonies compared with either treatment alone (Figure 1C and 1D). Collectively, AS602801 sensitized A2780 CSLC to carboplatin and paclitaxel.

AS602801 reduces survivin expression in a time-and dosedependent manner. Since the combination with AS602801 increased cell death of A2780 CSLC, we evaluated the effect of AS602801 on the expression of apoptosis-related proteins including pro-apoptotic proteins, pro-survival proteins, and proteins that belong to the inhibitor of apoptosis (IAP) family. Treatment with AS602801 markedly reduced the expression of survivin, while this treatment slightly reduced the expression of livin and BID. However, treatment with AS602801 did not alter the expression of the other proteins examined (Figure 2A). Since the alteration of the expression of survivin protein was the highest and because a reduction in survivin may explain the cell death increased by AS602801, we focused on survivin. Treatment with carboplatin or paclitaxel increased survivin expression, while AS602801 abolished such increase (Figure 2B). The reduction in survivin expression by AS602801 occurred in a time- and dose-dependent manner (Figure 2C and 2D).

Suppression of survivin expression sensitizes A2780 CSLCs to carboplatin and paclitaxel. To assess if the suppression of cell growth by AS602801 is mediated by a reduction in survivin expression, YM155, an inhibitor of survivin, was used as a treatment along with carboplatin or paclitaxel. The expression of survivin was reduced by treatment with YM155 (Figure 3A). The combination of YM155 with carboplatin or paclitaxel increased the number of dead cells and reduced cell viability compared with either drug alone (Figure 3B and 3C). To further confirm that the combinational effect was mediated by the reduction of survivin expression, survivin was knocked-down by two different siRNAs (siSurvivin \#1 and \#2). The expression of survivin was reduced by both siRNAs (Figure 3D). In the cells with survivin knockdown, the number of PI-positive (dead) cells was increased with and without carboplatin or paclitaxel treatment compared with control siRNA-treated cells (Figure 3E). The treatment of knockdown of survivin also reduced cell viability (Figure 3F). These results suggest that the suppression of survivin sensitizes A2780 CSLCs to carboplatin and paclitaxel.

AS602801 suppresses the expression of survivin in other types of CSCs and cancer cell lines. To determine if the reduction in survivin expression caused by AS602801 is a general phenomenon that also occurs in other types of CSCs and serum-cultured non-CSCs, several cell lines were treated with AS602801, and the alteration in survivin expression was examined. The expression of survivin was reduced in various types of CSCs: TOV-21G CSLC (an ovarian CSC line), PANC-1 CSLC (a pancreatic CSC line), A549 CLSC (a lung CSC line), GS-Y03 (a glioblastoma CSC line), and in various types of non-CSC cancer cell lines including TOV21G (an ovarian cancer cell line), A549, and H1299 (lung cancer cell lines) (Figure 4).

\section{Discussion}

CSCs comprise a rare population of cancer cells and have the properties of a high tumor initiation capacity and chemoresistance. Therefore, CSCs are sources of chemoresistant cancer cells in recurrent lesions after chemotherapy. We already reported that AS602801 inhibits tumor-initiating capacity (9) and metastatic potential (10) of CSCs, but the effect of AS602801 on the chemoresistance of CSCs was unknown. In this study, we showed that a combination of AS602801 with carboplatin or paclitaxel facilitated cell death and inhibited growth of the A2780 CSLC line compared with either AS602801, carboplatin, or paclitaxel alone. These results demonstrate that the addition of AS602801 chemosensitizes CSCs to standard chemotherapy. Since the safety of AS602801 has already been assessed in patients with endometriosis, AS602801 is a good candidate for combination with standard chemotherapy.

Survivin is a member of the IAP family and plays a role in the inhibition of apoptosis and the control of cell division (17, 18). Survivin is expressed during embryonic development, but its expression is lost in adult tissues except the testicular tissue, placental tissue, and bone marrow stem cells (19). Increased expression of survivin is observed in various types of cancer, and its increased expression is correlated with a poor prognosis (20-22). In CSCs of various types of cancer including ovarian cancer, the expression of survivin is elevated (23-27), and the suppression of survivin expression inhibits CSC-like properties $(25,27,28)$. Our data showed that survivin was reduced by treatment with AS602801 and that pharmacological and genetic suppression of survivin sensitized cancer cells to carboplatin and paclitaxel. These data suggest that chemosensitization by AS602801 might be mediated by the suppression of survivin. In agreement with 
A

Pro-apoptosis AS602801 - +

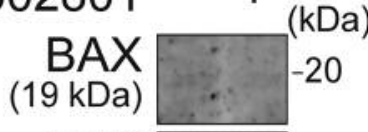

BAK

(28 kDa)

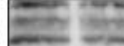

BOK

(18 kDa)

$-2-25$

BIM

$(12,15,23 \mathrm{kDa})$

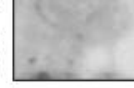

$-20$

$-25$

$-20$

$-15$

BAD
$(23 \mathrm{kDa})$
BID
$15,22 \mathrm{kDa})$ GAPDH
Pro-survival

AS602801 - +

PUMA

$(18,23 \mathrm{kDa})$

BCL-2

(26 kDa)

MCL-1

$(32,40 \mathrm{kDa})$

BCL-xL

$(29,34 \mathrm{kDa})$

GAPDH

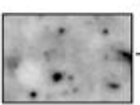

(kDa)
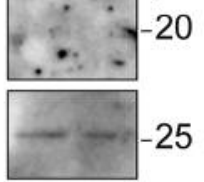

$-2=$

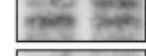

$-37$

$-37$

$-25$

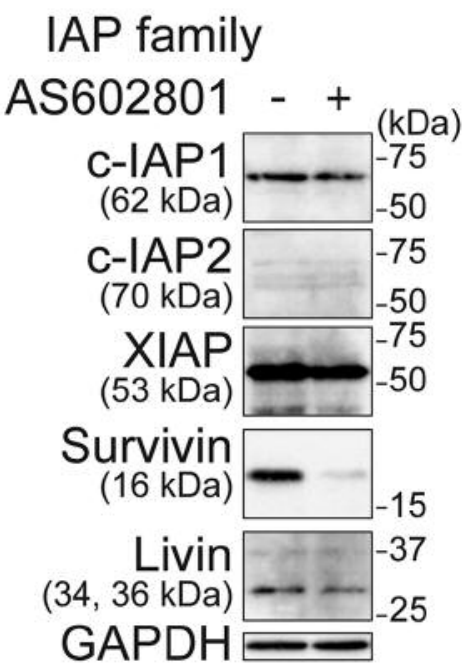

B
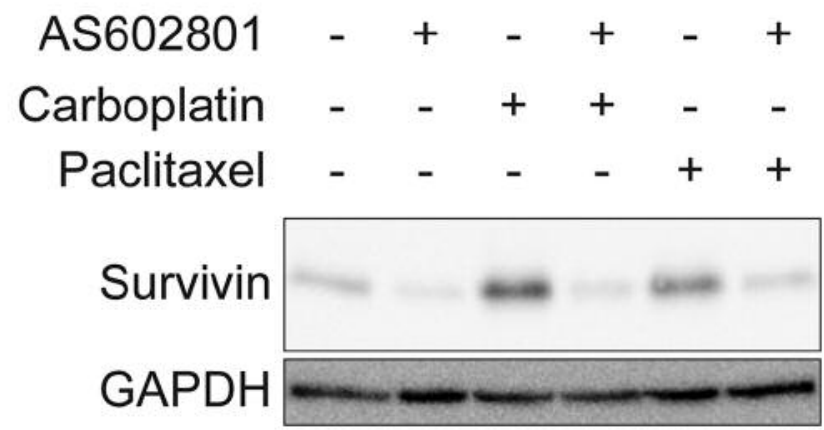

C

D

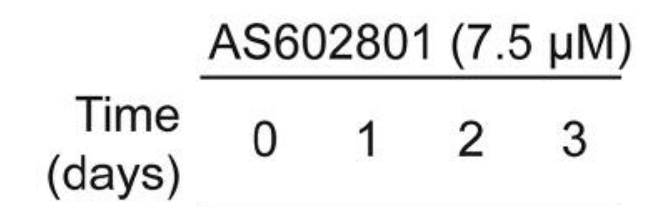

Survivin

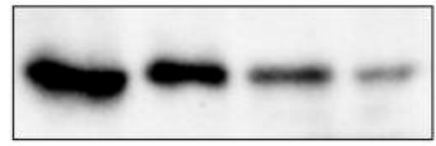

GAPDH

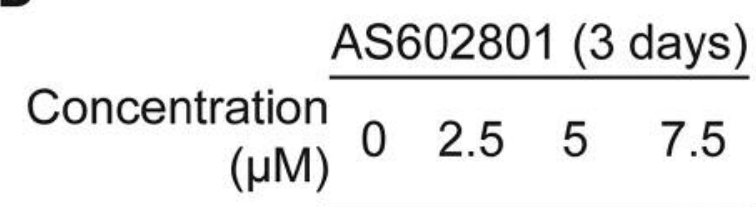

Survivin

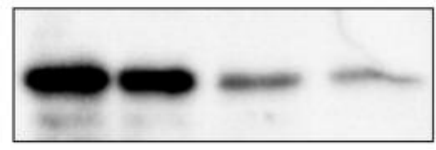

GAPDH

Figure 2. AS602801 reduces the expression of survivin in a time- and dose-dependent manner in A2780 CSLC cells. A2780 CSLC cells treated with or without AS602801 (7.5 $\mu$ M) for 3 days were subjected to western blot analysis of apoptosis-related proteins (A). The predicted molecular weight of the proteins is indicated in parentheses, and molecular weight markers are located on the right of the western blot images. A2780 CSLC cells treated with or without carboplatin $(5 \mu M)$ or paclitaxel $(2 \mathrm{nM})$ in the absence or presence of AS602801 at $7.5 \mu M$ for 3 days $(B)$, at $7.5 \mu M$ for different times $(C)$, and at the indicated concentrations for 3 days $(D)$. The cells were then subjected to western blot analysis of survivin expression. BCL-2: B-Cell lymphoma 2, BAX: BCL-2-associated X protein, BAK: BCL-2 antagonist/killer, BOK: BCL-2-related ovarian killer, BIM: BCL-2-like protein 11, BAD: BCL-2associated death promoter, BID: BH3 interacting-domain death agonist, PUMA: p53 upregulated modulator of apoptosis, MCL-1: myeloid cell leukemia1, c-IAP1: cellular inhibitor of apoptosis 1, XIAP: X-linked inhibitor of apoptosis protein, GAPDH: glyceraldehyde 3-phosphate dehydrogenase. 
A

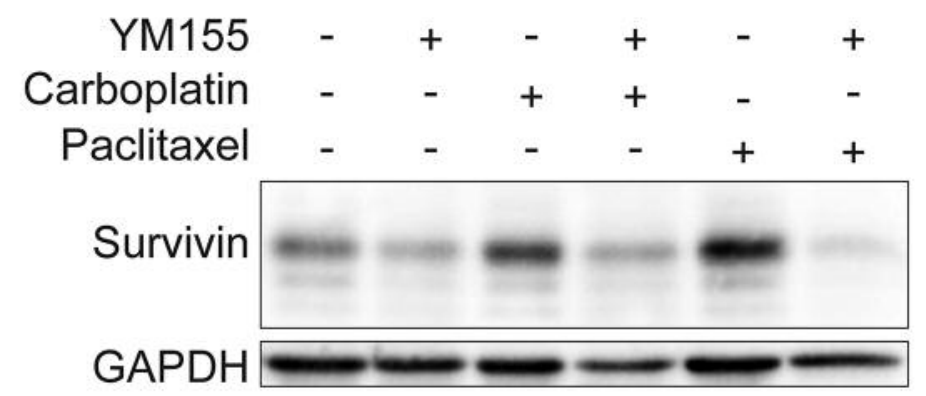

B

C

D
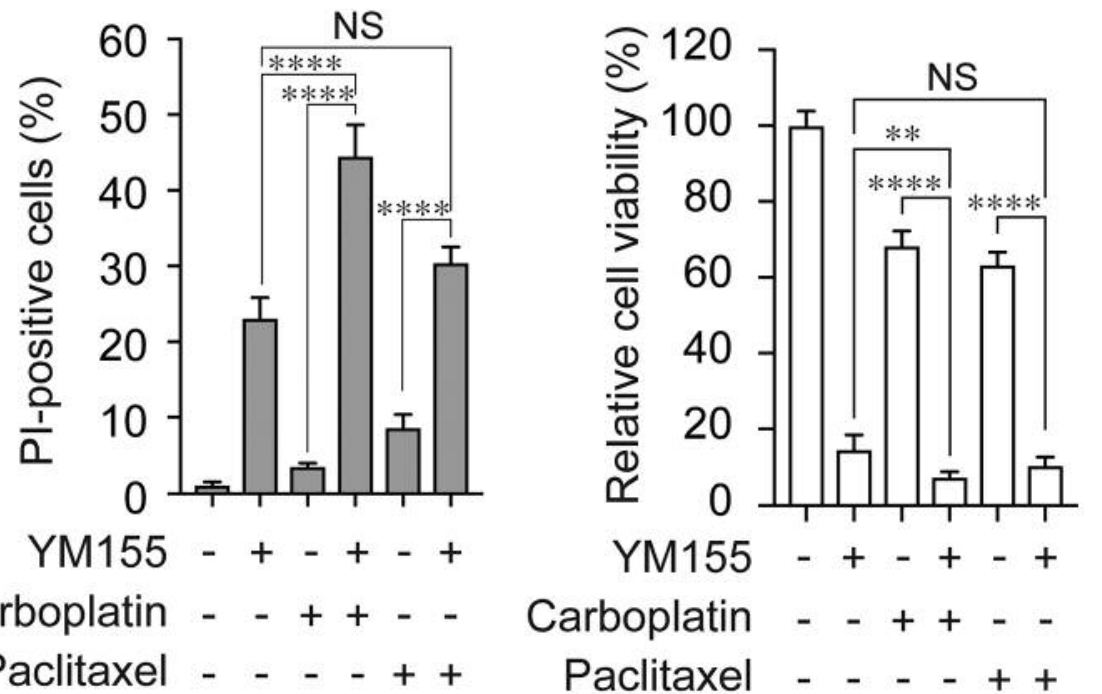

YM155 - + - + - +

GAPDH

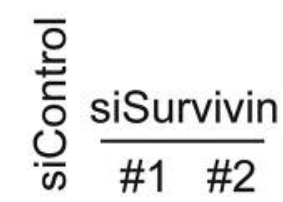

Carboplatin - - + + -

Paclitaxel - - - + +

E

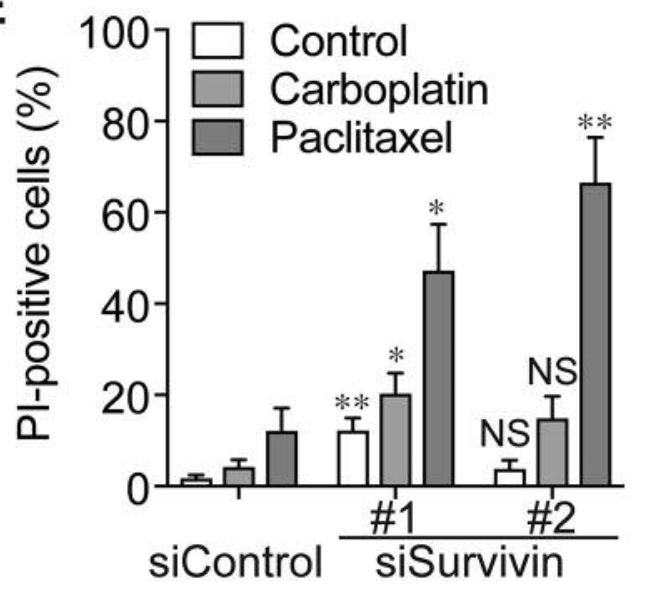

$\mathbf{F}$

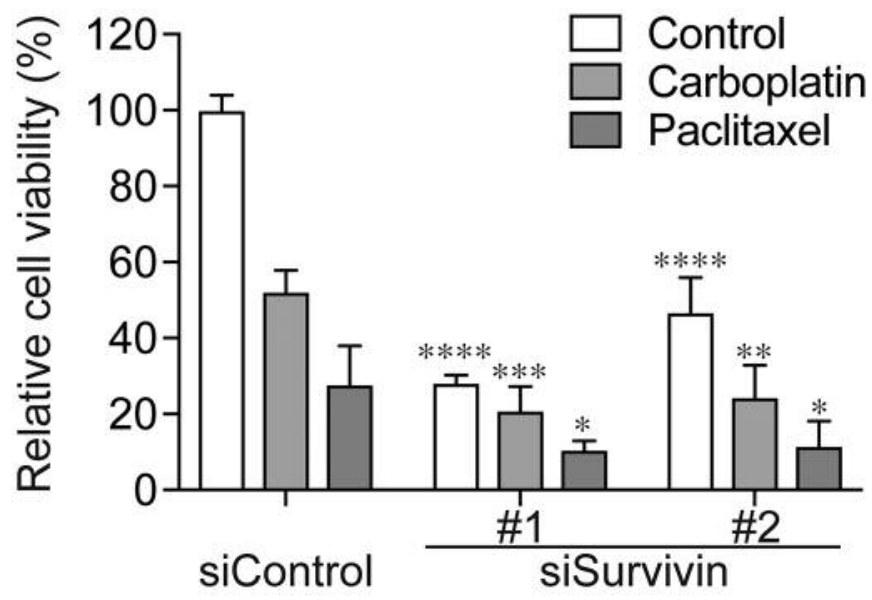

Figure 3. Pharmacological and genetic inhibition of survivin sensitizes A2780 CSLC cells to carboplatin and paclitaxel. A2780 CSLC cells treated with or without carboplatin $(5 \mu \mathrm{M})$ or paclitaxel $(2 \mathrm{nM})$ in the absence or presence of YM155 (10 nM) for 3 days were subjected to western blot analysis of survivin expression (A) or cell death assay using propidium iodide (PI) as a vital dye (B). A2780 CSLC cells treated with or without carboplatin (5 $\mu$ M) or paclitaxel $(2 \mathrm{nM})$ in the absence or presence of YM155 (10 nM) for 3 days were cultured for another 3 days in the absence of any drug. The cells were then subjected to a cell viability assay using WST-8 (C). A2780 CSLC cells were transfected with siRNAs against survivin (siSurvivin \#1 or \#2) or with a control RNA (siControl). The following day, the cells were treated with or without carboplatin $(5 \mu M)$ or paclitaxel $(2 n M)$ for 3 days and then subjected to immunoblot analysis of survivin $(D)$ or to a cell death assay using propidium iodide (PI) as a vital dye (E). To analyze the viability of cells, the cells were treated with or without carboplatin or paclitaxel for 3 days; they were then cultured for another 3 days without any drug, and then subjected to a WST-8 assay $(F)$. Values represent means $+S D$ from samples in four $(E$ and $F)$ or six $(B$ and $C)$ replicates of a representative experiment repeated with similar results. In B and C, NS: non-significant, $p>0.05$; significantly different: at $* * p<0.01$, and $* * * * p<0.0001$. In E and $F, N S p>0.05$; significantly different at: ${ }^{*}<<0.05, * * p<0.01, * * * p<0.001$, and $* * * * p<0.0001$ vs. the siControl. GAPDH: Glyceraldehyde 3-phosphate dehydrogenase. 


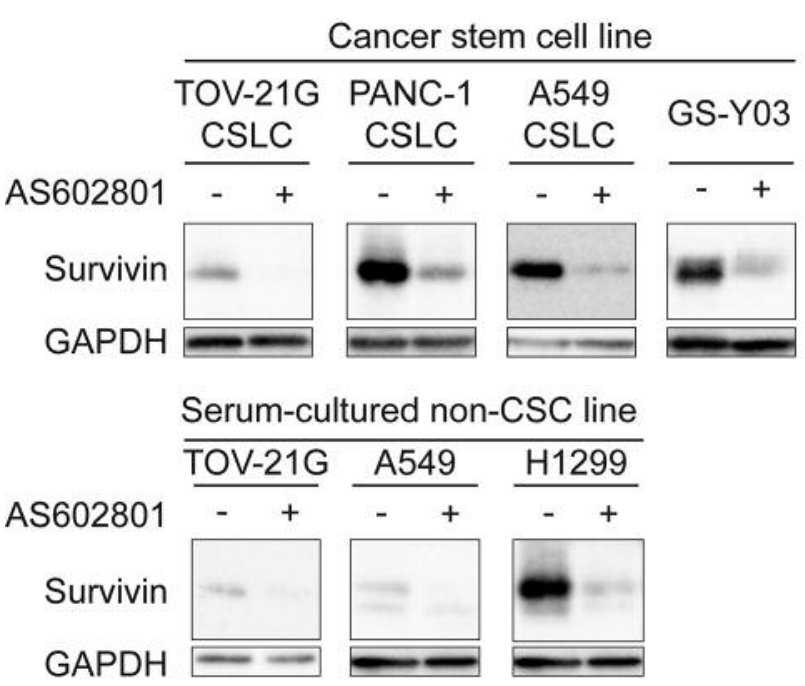

Figure 4. Suppression of survivin by AS602801 also occurs in other types of cancer stem cells (CSCs) and cancer cells. The indicated cell lines including CSCs and serum-cultured non-CSCs were treated with or without AS602801 (7.5 $\mu \mathrm{M})$ for 3 days and were then subjected to western blot analysis of survivin expression. GAPDH: Glyceraldehyde 3-phosphate dehydrogenase.

our observation, the inhibition of survivin was shown to sensitize CSCs to chemotherapeutic agents in colorectal (29), pancreatic (30), and breast (31) cancer. We showed that the expression of survivin was increased by treatment with both carboplatin and paclitaxel. It has been reported that irinotecan and hydroxyurea induced the expression of survivin, whereas oxaliplatin reduced the expression of survivin in HCT116 colon cancer cells (32). The induction of survivin is differentially regulated by chemotherapeutic agents. A combination of agents that suppresses survivin, such as AS602801, and chemotherapeutic agents that induce expression of survivin can be an efficacious combination therapy against cancer cells, because A602801 attenuates the chemoresistance that results from high levels of survivin. Since our data indicated that the expression of survivin was suppressed by AS602801 in other types of CSCs and cancer cells, AS602801 is expected to be effective, not only for ovarian CSCs, but also for other types of CSCs and non-CSCs.

In this study, we discovered a new role for AS602801: chemosensitization of CSCs, in addition to the previously known roles of AS602801: suppression of tumor initiation capacity and metastasis. Our results imply that the inclusion of AS602801 in a standard chemotherapy regimen might improve prognosis of cancer including ovarian cancer by enhancing the cytotoxicity of chemotherapeutic agents in CSCs.

\section{Conflicts of Interest}

The Authors declare no conflict of interest in regard to this study.

\section{Acknowledgements}

This work was supported by Grants-in-Aid for Scientific Research, and for Young Scientists from the Ministry of Education, Culture, Sports, Science and Technology of Japan.

\section{References}

1 Beck B and Blanpain C: Unravelling cancer stem cell potential. Nat Rev Cancer 13: 727-738, 2013.

2 Maugeri-Sacca M, Vigneri P and De Maria R: Cancer stem cells and chemosensitivity. Clin Cancer Res 17: 4942-4947, 2011.

3 Heintz AP, Odicino F, Maisonneuve P, Quinn MA, Benedet JL, Creasman WT, Ngan HY, Pecorelli S and Beller U: Carcinoma of the ovary. FIGO 26th Annual Report on the Results of Treatment in Gynecological Cancer. Int J Gynaecol Obstet 95(Suppl 1): S161-192, 2006.

4 Monk BJ and Coleman RL: Changing the paradigm in the treatment of platinum-sensitive recurrent ovarian cancer: From platinum doublets to nonplatinum doublets and adding antiangiogenesis compounds. Int J Gynecol Cancer 19(Suppl 2): S63-67, 2009.

5 Cannistra SA: Cancer of the ovary. N Engl J Med 351: 25192529, 2004

6 Abubaker K, Latifi A, Luwor R, Nazaretian S, Zhu H, Quinn MA, Thompson EW, Findlay JK and Ahmed N: Short-term single treatment of chemotherapy results in the enrichment of ovarian cancer stem cell-like cells leading to an increased tumor burden. Mol Cancer 12: 24, 2013.

7 Ahmed N, Abubaker K, Findlay J and Quinn M: Cancerous ovarian stem cells: obscure targets for therapy but relevant to chemoresistance. J Cell Biochem 114: 21-34, 2013.

8 Wang T, Shigdar S, Gantier MP, Hou Y, Wang L, Li Y, Shamaileh HA, Yin W, Zhou SF, Zhao X and Duan W: Cancer stem cell targeted therapy: progress amid controversies. Oncotarget 6: 44191-44206, 2015.

9 Okada M, Kuramoto K, Takeda H, Watarai H, Sakaki H, Seino S, Seino M, Suzuki S and Kitanaka C: The novel JNK inhibitor AS602801 inhibits cancer stem cells in vitro and in vivo. Oncotarget 7: 27021-27032, 2016.

10 Kuramoto K, Yamamoto M, Suzuki S, Sanomachi T, Togashi K, Seino S, Kitanaka C and Okada M: AS602801, an anti-cancer stem cell drug candidate, suppresses gap-junction communication between lung cancer stem cells and astrocytes. Anticancer Res 38: 5093-5099, 2018.

11 Togashi K, Okada M, Yamamoto M, Suzuki S, Sanomachi T, Seino S, Yamashita H and Kitanaka C: A small-molecule kinase inhibitor, CEP-1347, inhibits survivin expression and sensitizes ovarian cancer stem cells to paclitaxel. Anticancer Res 38: 45354542, 2018.

12 Seino M, Okada M, Shibuya K, Seino S, Suzuki S, Takeda H, Ohta T, Kurachi $\mathrm{H}$ and Kitanaka C: Differential contribution of ROS to resveratrol-induced cell death and loss of self-renewal capacity of ovarian cancer stem cells. Anticancer Res 35: 85-96, 2015. 
13 Seino M, Okada M, Shibuya K, Seino S, Suzuki S, Ohta T, Kurachi $\mathrm{H}$ and Kitanaka C: Requirement of JNK signaling for self-renewal and tumor-initiating capacity of ovarian cancer stem cells. Anticancer Res 34: 4723-4731, 2014.

14 Sakaki H, Okada M, Kuramoto K, Takeda H, Watarai H, Suzuki S, Seino S, Seino M, Ohta T, Nagase S, Kurachi H and Kitanaka C: GSKJ4, a selective Jumonji H3K27 demethylase inhibitor, effectively targets ovarian cancer stem cells. Anticancer Res 35: 6607-6614, 2015.

15 Okada M, Takeda H, Sakaki H, Kuramoto K, Suzuki S, Sanomachi T, Togashi K, Seino S and Kitanaka C: Repositioning CEP-1347, a chemical agent originally developed for the treatment of Parkinson's disease, as an anti-cancer stem cell drug. Oncotarget 8: 94872-94882, 2017.

16 Seino M, Okada M, Sakaki H, Takeda H, Watarai H, Suzuki S, Seino S, Kuramoto K, Ohta T, Nagase S, Kurachi $H$ and Kitanaka C: Time-staggered inhibition of JNK effectively sensitizes chemoresistant ovarian cancer cells to cisplatin and paclitaxel. Oncol Rep 35: 593-601, 2016.

17 Altieri DC and Marchisio PC: Survivin apoptosis: An interloper between cell death and cell proliferation in cancer. Lab Invest 79: 1327-1333, 1999.

18 Ryan BM, O’Donovan N and Duffy MJ: Survivin: A new target for anticancer therapy. Cancer Treat Rev 35: 553-562, 2009.

19 Fukuda S and Pelus LM: Regulation of the inhibitor-of-apoptosis family member survivin in normal cord blood and bone marrow CD34(+) cells by hematopoietic growth factors: Implication of survivin expression in normal hematopoiesis. Blood 98: 20912100, 2001.

20 Cohen C, Lohmann CM, Cotsonis G, Lawson D and Santoianni R: Survivin expression in ovarian carcinoma: correlation with apoptotic markers and prognosis. Mod Pathol 16: 574-583, 2003.

$21 \mathrm{Xu} \mathrm{C}$, Yamamoto-Ibusuki M, Yamamoto Y, Yamamoto S, Fujiwara S, Murakami K, Okumura Y, Yamaguchi L, Fujiki Y and Iwase H: High survivin mRNA expression is a predictor of poor prognosis in breast cancer: A comparative study at the mRNA and protein level. Breast Cancer 21: 482-490, 2012.

22 Cerda-Opazo P, Valenzuela-Valderrama M, Wichmann I, Rodriguez A, Contreras-Reyes D, Fernandez EA, CarrascoAvino G, Corvalan AH and Quest AFG: Inverse expression of survivin and reprimo correlates with poor patient prognosis in gastric cancer. Oncotarget 9: 12853-12867, 2018.

23 Siddharth S, Das S, Nayak A and Kundu CN: Survivin as a marker for quiescent-breast cancer stem cells - An intermediate, adherent, pre-requisite phase of breast cancer metastasis. Clin Exp Metastasis 33: 661-675, 2016.

24 Dong Z, Yang L and Lai D: KLF5 strengthens drug resistance of ovarian cancer stem-like cells by regulating survivin expression. Cell Prolif 46: 425-435, 2013.
25 Dahan P, Martinez Gala J, Delmas C, Monferran S, Malric L, Zentkowski D, Lubrano V, Toulas C, Cohen-Jonathan Moyal E and Lemarie A: Ionizing radiations sustain glioblastoma cell dedifferentiation to a stem-like phenotype through survivin: possible involvement in radioresistance. Cell Death Dis 5: e1543, 2014.

26 Carter BZ, Qiu Y, Huang X, Diao L, Zhang N, Coombes KR, Mak DH, Konopleva M, Cortes J, Kantarjian HM, Mills GB, Andreeff $M$ and Kornblau SM: Survivin is highly expressed in CD34(+)38(-) leukemic stem/progenitor cells and predicts poor clinical outcomes in AML. Blood 120: 173-180, 2012.

27 Wang J, Liu Z, Zhang D, Liu R, Lin Q, Liu J, Yang Z, Ma Q, Sun D, Zhou X and Jiang G: FL118, a novel survivin inhibitor, wins the battle against drug-resistant and metastatic lung cancers through inhibition of cancer stem cell-like properties. Am J Transl Res 9: 3676-3686, 2017.

28 Guvenc H, Pavlyukov MS, Joshi K, Kurt H, BanasavadiSiddegowda YK, Mao P, Hong C, Yamada R, Kwon CH, Bhasin D, Chettiar S, Kitange G, Park IH, Sarkaria JN, Li C, Shakhparonov MI and Nakano I: Impairment of glioma stem cell survival and growth by a novel inhibitor for survivin-ran protein complex. Clin Cancer Res 19: 631-642, 2013.

29 AlShamaileh H, Wang T, Xiang D, Yin W, Tran PH, Barrero RA, Zhang PZ, Li Y, Kong L, Liu K, Zhou SF, Hou Y, Shigdar S and Duan W: Aptamer-mediated survivin RNAi enables 5fluorouracil to eliminate colorectal cancer stem cells. Sci Rep 7: 5898,2017

30 Takeda H, Okada M, Suzuki S, Kuramoto K, Sakaki H, Watarai H, Sanomachi T, Seino S, Yoshioka T and Kitanaka C: Rhoassociated protein kinase (ROCK) inhibitors inhibit survivin expression and sensitize pancreatic cancer stem cells to gemcitabine. Anticancer Res 36: 6311-6318, 2016.

31 Wang T, Gantier MP, Xiang D, Bean AG, Bruce M, Zhou SF, Khasraw M, Ward A, Wang L, Wei MQ, AlShamaileh H, Chen L, She X, Lin J, Kong L, Shigdar S and Duan W: EpCAM Aptamer-mediated survivin silencing sensitized cancer stem cells to doxorubicin in a breast cancer model. Theranostics 5: 14561472, 2015.

32 Rauch A, Carlstedt A, Emmerich C, Mustafa AM, Goder A, Knauer SK, Linnebacher M, Heinzel $\mathrm{T}$ and Kramer $\mathrm{OH}$ : Survivin antagonizes chemotherapy-induced cell death of colorectal cancer cells. Oncotarget 9: 27835-27850, 2018.

Received October 22, 2018

Revised November 1, 2018

Accepted November 2, 2018 